\title{
Numbers of "blobs" in the primary visual cortex of neonatal and adult monkeys
}

\author{
(development/primate/brain/vision)
}

Dale Purves* and Anthony-Samuel laMantia*

Department of Anatomy and Neurobiology, Washington University School of Medicine, 660 South Euclid Avenue, Saint Louis, MO 63110

Contributed by Dale Purves, May 14, 1990

\begin{abstract}
We have examined the number of "blobs" (cytochrome oxidase-positive cortical modules) in the primary visual cortex (area 17) of infant and adult rhesus monkeys. The density of these iterated circuits-about five per $\mathbf{~ m m}^{2}$ - was not significantly different in three newborn and three mature animals. Measurement of the surface of area 17 in serial sections, however, showed that the total area occupied by the primary visual cortex increases by about $50 \%$ during maturation. Based on these measurements, the number of blobs in this species is about 8000 at birth and about 12,000 in maturity. Evidently, these complex functional units are added gradually to the developing primate brain over a period that extends into postnatal life.
\end{abstract}

The mammalian brain grows substantially during postnatal life. In man, for example, brain weight increases about 4-fold between birth and the 20th year (1). Although brain growth during late (postnatal) stages of mammalian development is universal, the consequences of this phenomenon for the neural circuitry of the brain are unclear. Neural circuitry and brain growth could be related in one of three ways. (i) The number of neural circuits might be determined quite early and thus remain stable during the postnatal enlargement of the brain. (ii) The number of neural circuits might decrease as the brain continues to grow, perhaps as a result of experiencedependent selection. (iii) The number of neural circuits might increase during postnatal life and, therefore, contribute to the growth of the brain. The relationship between brain growth and neural circuitry has not been resolved because of the difficulty of counting circuits in the mammalian brain.

In the present report, we make use of the fact that several regions of the primate cerebral cortex are characterized by iterated circuits or "modules" (for review, see ref. 2). Cortical modules were first recognized during electrophysiological analysis of the somatosensory and visual cortices (3-5). Subsequently, modules have been discerned anatomically by virtue of afferent-terminal segregation (6-9) or discontinuous staining after application of various histochemical markers (10-12). Because some classes of modules can be visualized and counted, it is possible to address quantitatively the relationship between brain growth and the number of constituent neural circuits. Here we have examined a particularly accessible type of module, cytochrome oxidase-positive "blobs" in the primary visual cortex of monkeys. Blobs are repeating units of increased cytochrome oxidase reactivity found in the striate cortex of most, and probably all, primates (11-16). In some species, their constituent cells are particularly responsive to color and project in a specific manner to extrastriate visual cortex $(15,17)$. Our initial results in an ongoing study of cortical development

The publication costs of this article were defrayed in part by page charge payment. This article must therefore be hereby marked "advertisement" in accordance with 18 U.S.C. $\$ 1734$ solely to indicate this fact. indicate that these modules are gradually added to the developing brain.

\section{METHODS AND MATERIALS}

Six male rhesus monkeys (Macaca mulatta), three neonatal and three adult, were sedated with ketamine $(10 \mathrm{mg} / \mathrm{kg})$ and deeply anesthetized with nembutal $(100 \mathrm{mg} / \mathrm{kg})$. The animals were then perfused transcardially with isotonic phosphatebuffered saline at $4^{\circ} \mathrm{C}$ followed by an ice-cold solution of $0.5 \%$ glutaraldehyde and $2.5 \%$ (wt/vol) paraformaldehyde in phosphate buffer. The fixative was immediately rinsed from the tissues by further perfusion with ice-cold phosphate-buffered saline. After perfusion, the brains were removed from the skull, weighed, and photographed. In the left hemisphere of each animal, we measured blob density after visualizing these units by means of cytochrome oxidase histochemistry (18); in the right (contralateral) hemisphere we measured the surface area of the primary visual cortex.

The primary visual cortex of the left occipital lobe was dissected from both the opercular and calcarine regions and equilibrated with a series of buffered sucrose solutions [from $10 \%$ to $30 \%$ (wt/vol)] for cryoprotection. These pieces were then flattened by compressing them between two Saranwrapped slides, and frozen on a metal surface cooled to $-20^{\circ} \mathrm{C}$. Subsequently, the frozen tissue was placed in cryoembedding medium and immersed in 2-methylbutane cooled to the temperature of liquid nitrogen. The cortex was then sectioned tangentially at $50 \mu \mathrm{m}$ in a cryostat and mounted on gelatin-coated slides. The sections were air-dried for $30 \mathrm{~min}$ and incubated in the dark for $2-4 \mathrm{hr}$ at $37^{\circ} \mathrm{C}$ in a phosphatebuffered solution of diaminobenzidine $(0.5 \mathrm{mg} / \mathrm{ml})$ to which sucrose $(0.04 \mathrm{~g} / \mathrm{ml})$, catalase $(0.2 \mathrm{mg} / \mathrm{ml})$, and cytochrome $c$ $(0.2 \mathrm{mg} / \mathrm{ml})$ had been added (18). When the histochemical reaction was judged complete by visual inspection, the reaction was stopped by repeated washings in phosphate buffer. The sections were then cleared, a coverslip was mounted, and slides were examined in the light microscope at a total magnification of $\times 18.5$. In these tangentially cut, cytochrome oxidase-stained sections of the left hemisphere, we determined the areal density of blobs in cortical layers II and III by tracing them with the aid of a camera lucida in 10 regions of the operculum. Each area sampled measured 7-15 $\mathrm{mm}^{2}$ and contained 20-90 blobs.

The contralateral (right) hemisphere of each animal was divided into three blocks for serial coronal sectioning and measurement of the area occupied by the primary visual cortex (Brodmann's area 17). The blocks were infiltrated with sucrose over a period of 1-2 weeks, frozen in dry ice, set in cryoembedding medium, and sectioned at $50 \mu \mathrm{m}$ in a cryostat. Every 10th section was mounted directly on gelatincoated slides and stained with cresyl violet, and the linear

\footnotetext{
*Present address: Department of Neurobiology, Box 3209, Duke University Medical Center, Durham, NC 27710.
} 

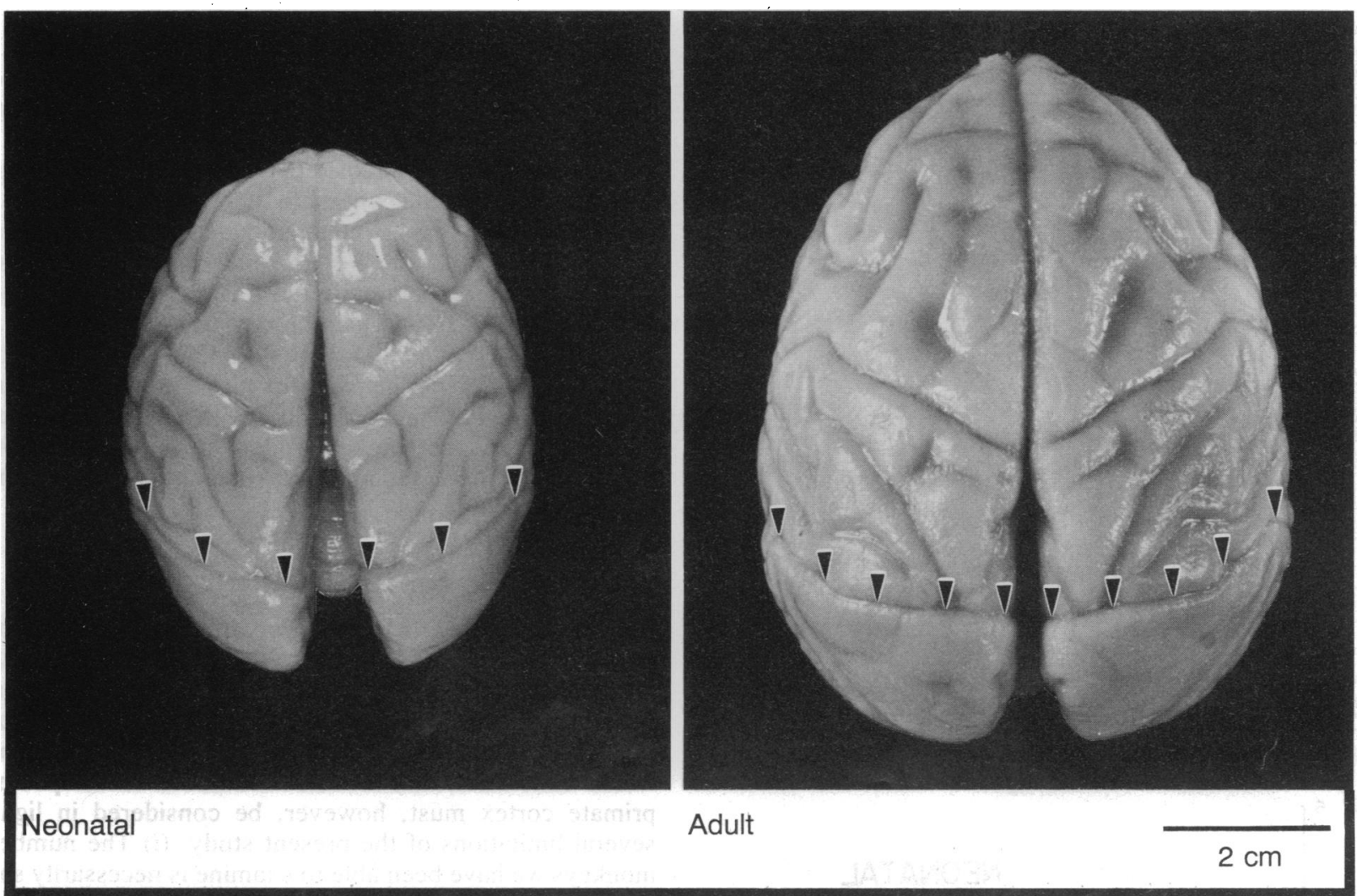

Fig. 1. Dorsal view of a rhesus monkey brain at birth (Left) and in maturity (Right). The exposed portion of the striate cortex lies posterior to the lunate sulcus (arrowheads); the buried (calcarine) portion cannot be seen without dissection. The overall size of the brain and the apparent size of the portion of the visual cortex that can be seen in this view are larger in the adult.

extent of area 17 at the cortical surface of each section was measured. The surface area of the primary visual cortex was calculated from the following equation (19):

$$
S=s\left[\frac{1}{2}\left(Y_{1}+Y_{n+1}\right)+Y_{2}+Y_{3}+\ldots+Y_{n}\right] \text {, }
$$

where $S$ is the surface area, $s$ the interval between the sections examined $(0.5 \mathrm{~mm})$, and $Y_{1}, Y_{2}, Y_{3}, \ldots Y_{n}$ are the surface lengths of area 17 in the individual sections examined.

Finally, the number of blobs in the brain of each animal was determined by multiplying the surface area of the primary visual cortex determined in the right hemisphere by the average density of blobs measured in the left hemisphere. The total number of blobs was assumed to be double the value in one hemisphere.

\section{RESULTS}

The overall size and weight of the three neonatal monkey brains that we examined were substantially less than the size and weight of three adult brains prepared in the same way (Fig. 1 and Table 1). This difference was also apparent upon examination of the primary visual cortex. Thus the exposed (opercular) region, which is easily identified in the rhesus monkey, was smaller in the neonates than in the adults (Fig. 1). The depth and length of the calcarine fissure were also less in the neonatal animals, suggesting that the buried portion of the visual cortex, as the opercular region, is smaller at birth than in maturity.

To verify these observations, we cut serial sections through the occipito-parietal region of the right hemisphere of each brain and measured the extent of area 17. The range of surface area values calculated from these measurements in

Table 1. Numbers of blobs in the primary visual cortex of neonatal and adult rhesus monkeys

\begin{tabular}{lccccc}
\hline Animal & Age & $\begin{array}{c}\text { Brain weight, } \\
\mathrm{g}\end{array}$ & $\begin{array}{c}\text { Area of } \\
\text { striate } \\
\text { cortex, } \\
\mathrm{mm}^{2}\end{array}$ & $\begin{array}{c}\text { Opercular } \\
\text { blob } \\
\text { density, no. } \\
\text { per } \mathrm{mm}^{2}\end{array}$ & $\begin{array}{c}\text { Overall } \\
\text { number } \\
\text { of blobs per } \\
\text { hemisphere }\end{array}$ \\
\hline Neonatal & 3 days & 50 & 670 & 4.6 & 3082 \\
& 4 days & 50 & 825 & 6.0 & 4950 \\
& 5 days & 64 & 910 & 5.0 & 4550 \\
Adult & 7 years & 106 & 1210 & 4.2 & $4194 \pm 568$ (mean \pm SEM) \\
& 8 years & 105 & 1148 & 5.8 & 5082 \\
& 12 years & 101 & 1246 & 4.9 & 6658 \\
& & & & & $5948 \pm 462$ (mean \pm SEM)*
\end{tabular}

*Despite the small sample size, the difference between the mean values of neonatal and adult animals is significant $(P=0.0373)$. 

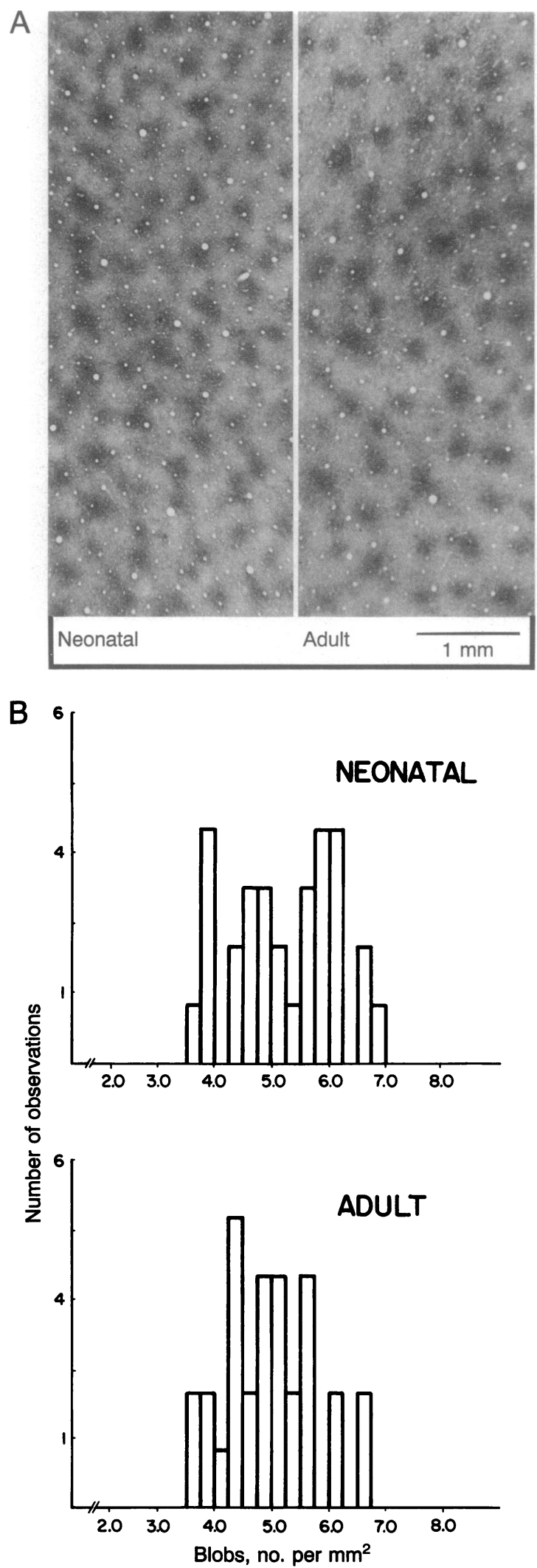

Fig. 2. Density of cytochrome oxidase-positive blobs in the primary visual cortex of neonatal and adult rhesus monkeys. $(A)$ Blobs in the mid-opercular region of the striate cortex of a newborn monkey (Left) and an adult (Right). (B) Distribution of density measurements in neonatal and adult animals (see also Table 1). There is no significant difference between the two distributions. Blob densities measured in the calcarine cortex were similar to those in the operculum. the infant monkeys was $670-910 \mathrm{~mm}^{2}$, with a mean of 810 $\mathrm{mm}^{2}$. The range in the three adult animals was 1148 to 1246 $\mathrm{mm}^{2}$, with a mean of $1201 \mathrm{~mm}^{2}$. Thus, the area occupied by the primary visual cortex increases by about $50 \%$ during the postnatal maturation of this species (see also ref. 20).

The density of blobs in the opercular cortex of each animal was determined in the contralateral hemisphere (Fig. 2). The range of densities in the infant animals was 3.7-6.8 per $\mathrm{mm}^{2}$ and in the adults was 3.5-6.7 per $\mathrm{mm}^{2}$. There was no significant difference among the measured densities in the two groups (Fig. $2 B$ and Table 1). Based on these values, the calculated number of blobs in the infant monkeys ranged from 3082 to 4950 per hemisphere with a mean of 4194 units; values in the adult were 5082-6658 with a mean of 5948 units (Table 1). Thus the number of blobs in the rhesus monkey visual cortex increases by approximately $40 \%$ during postnatal maturation.

\section{DISCUSSION}

We have found that there are approximately 8000 blobs in the primary visual cortex of newborn rhesus monkeys and 12,000 in adults. Apparently, the number of neural circuits increases in this part of the growing brain, rather than remaining (stable or declining because of selective or regressive processes).

Our conclusion that modules are added to the postnatal primate cortex must, however, be considered in light of several limitations of the present study. (i) The number of monkeys we have been able to examine is necessarily small. (ii) Blobs are aptly named. Whereas these circuits are more easily visualized and counted than other cortical units, such as ocular dominance or orientation columns, blobs are not always distinct from one another (see Fig. 2A). Accordingly, a decision must sometimes be made about whether a cytochrome oxidase-positive patch represents one blob or two. (iii) Another problem is the difficulty of measuring the surface area of the visual cortex (or any cortical area) from serial sections (cf. refs. 20 and 21). These limitations, which are inherent in our analysis, argue for some caution until a larger sample of rhesus monkeys and other primate species can be examined.

If a more extensive study confirms that the postnatal addition of complex circuits is a feature of the developing mammalian brain, this process will have various implications for understanding several poorly understood aspects of neural development (see also refs. 22 and 23). These include the prevalence of critical periods, the enhanced ability of infant brains to recover from injury, and the remarkable ability of maturing brains to permanently store large amounts of new information. Each of these phenomena may depend upon the prolonged construction of complex neural circuitry in postnatal life.

We are grateful to Pat Newton for her assistance and to Josh Sanes and Nigel Daw for useful comments on the manuscript.

1. Dekaban, A. S. \& Sadowsky, D. (1978) Ann. Neurol. 4, 345.

2. Mountcastle, V. B. (1978) in The Mindful Brain: Cortical Organization and the Group-Selective Theory of Higher Brain Function, eds. Edelman, G. M. \& Mountcastle, V. B. (MIT Press, Cambridge, MA), pp. 7-50.

3. Mountcastle, V. B. (1957) J. Neurophysiol. 20, 408-434.

4. Hubel, D. H. \& Wiesel, T. N. (1963) J. Physiol. 165, 559-568.

5. Hubel, D. H. \& Wiesel, T. N. (1968) J. Physiol. 195, 215-243.

6. Hubel, D. H., Wiesel, T. N. \& LeVay, S. (1977) Philos. Trans. $R$. Soc. London B 278, 377-409.

7. Jones, E. G., Burton, H. \& Porter, R. (1975) Science 190, 572-574. 
8. Goldman, P. S. \& Nauta, W. J. H. (1977) Brain Res. 122, 393-413.

9. Livingstone, M. S. \& Hubel, D. H. (1982) Proc. Natl. Acad. Sci. USA 79, 6098-6101.

10. Horton, J. C. \& Hubel, D. H. (1981) Nature (London) 292, 762-764.

11. Horton, J. C. (1984) Philos. Trans. R. Soc. London B 304, 199-272.

12. Hendrickson, A. E. (1985) Trends Neurosci. 7, 406-410.

13. Carroll, E. W. \& Wong-Riley, M. T. T. (1984) J. Comp. Neurol. 222, 1-17.

14. Horton, J. C. \& Hedley-Whyte, E. T. (1984) Philos. Trans. $R$. Soc. London B 304, 255-272.

15. Livingstone, M. S. \& Hubel, D. S. (1984) J. Neurosci. 4, 309-356.
16. Condo, G. \& Casagrande, V. A. (1990) J. Comp. Neurol. 293, 632-645.

17. Livingstone, M. S. \& Hubel, D. S. (1987) J. Neurosci. 7, 3371-3377.

18. Wong-Riley, M. (1979) Brain Res. 171, 11-28.

19. Tuttle, L. \& Satterly, J. (1925) Theory of Measurement (Longmans, London), Chap. 22, pp. 290-297.

20. Gottlieb, M. D., Pasik, P. \& Pasik, T. (1985) Exp. Brain Res. 17, 53-62.

21. O'Kusky, J. \& Colonnier, M. (1982) J. Comp. Neurol. 210, 291-306.

22. LaMantia, A.-S. \& Purves, D. (1989) Nature (London) 341, 646-649.

23. Pomeroy, S. L., LaMantia, A.-S. \& Purves, D. (1990) J. Neurosci.10, 1952-1966. 